\title{
Editorial
}

\section{Improving ventricular systolic function: simple messages from complex models}

The useful work done by the left ventricle is the generation of pressure and flow within the circulation. While pressure and flow are accessible concepts, the models used to describe the ventricular mechanics that are responsible for pressure and flow are increasingly obscure. In a recent issue of Heart, Popovic and colleagues describe some simple observations about changes in ventricular pressures and volumes before and after the Batista procedure. ${ }^{1}$ The message is simple but expressed in the language of modern ventricular mechanics, it is difficult to disentangle.

In isolated heart muscle preparations the relations between tension and length, and shortening velocity and length, are well known. Attempts to describe the contractile performance in the intact left ventricle in vivo extrapolate these relations, substituting intracavity pressure for tension and volume change for length change. Indices of contractile function have thus been described from analysis of pressure-time relations ( $\mathrm{dp} / \mathrm{dt} \max$ ), or dimension-time relations (Vmax). Apart from the technical difficulties associated with actually measuring or deriving these indices, they are of limited value for theoretical reasons. Ventricular systolic function involves changes in both pressure and volume; models that assess only one of these parameters are both incomplete and, crucially, dependent on the prevailing loading conditions of the heart.

The time varying elastance model of ventricular function ${ }^{2}$ has been an advance, describing ventricular contractile performance in terms of the relations between pressure and volume during the cardiac cycle. This model defines the elastance of the ventricle at any instant in the cycle as the instantaneous pressure divided by volume. The elastance is greatest at the end of ventricular ejection and in a pure sense can be taken to define end systole. Under different loading conditions values of maximal elastance form a straight line on a graph of pressure as a function of volume, and the gradient of this relation ( $\mathrm{E} \max$ ) is interpreted as a load independent measure of contractile function. The gradient is sensitive to changes in inotropic state; the steeper the gradient the greater the ventricular contractility.

The pump function of the ventricle is determined not only by its contractility but by the characteristics of the vascular bed into which it ejects. The fluid dynamic characteristics of the arterial tree-the left ventricular afterload - are impossibly complex to describe mathematically. A useful approximation, however, is to derive an arterial elastance $(\mathrm{Ea})$ which is simply systolic pressure divided by the volume accommodated during a single ejection (the ventricular stroke volume). ${ }^{3}$ One of the successes of the elastance model of ventricular function is the description of ventricular systolic function and the ventricular afterload in the same terms. The ratio between arterial and ventricular elastance is interpreted as an index of the coupling between the ventricle and its afterload, and an increase in this ratio is associated with increased efficiency of this coupling.

There are many technical problems in the measurement of simultaneous ventricular pressure and volume in human subjects. Some of these are evident in Popovic's study. ${ }^{1}$ For example, femoral pressure is used as a surrogate for intraventricular pressure, and the end systolic volume is simply assessed as the smallest measured volume extrapolated from single plane angiography; values of maximal elastance defined in this way are made at only two different loading conditions and the linear regression analysis employed to derive end systolic elastance (an approximation to E max), is thus based on only two points. The actual values of elastance reported must therefore be interpreted with extreme caution. However, the uniform observation that partial left ventriculotomy was associated with an increase in the value does suggest something about the overall effect of the procedure on ventricular contractile function.

In simple terms they have shown that the ventricular volumes are smaller after the procedure but that there is no change in the effective arterial elastance, and that therefore systolic pressure and stroke volume are unaffected. The loading studies suggest that the increase in systolic pressure that occurs in the ventricle with an increase in its filling is greater after the procedure, and this can be interpreted by analogy with Starling's law as an improvement in contractility. Thus, after the procedure the same useful pump function is performed by a smaller ventricle with improved contractile function. This is probably a good thing although we are now well aware that interventions that improve ventricular performance have not always led to an improvement in the clinical end points of heart failure associated with left ventricular dysfunction.

Ventricular systolic function is more complex than the contractile behaviour of isolated heart muscle. Simple relations between ventricular pressure and volume can never accurately reflect the effects of regional inhomogeneity and ventricular incoordination. However, it is sometimes useful to think of the heart as a black box generating pressure and flow in the circulation, and this way of thinking suggests that the Batista procedure may have something to offer.

A J BISHOP

North Hampshire Hospital, Aldermaston Road

Basingstoke, Hampshire RG24 9NA, UK

\footnotetext{
1 Popovic Z, Miric M, Gradinac S, et al. Partial left ventriculectomy improves left ventricular and systolic elastance in patients with idiopathic dilated cardiomyopathy. Heart 2000;83:316-19.

2 Suga H, Sagawa K, Shoukas A. Load independence of the instantaneous pressure volume ratio of the canine left ventricle and effects of epinephrine and heart rate on the ratio. Circ Res 1973;32:314-22.

3 Kelly P, Ting C, Yang T, et al. Effective arterial elastance as index of arterial vascular load in humans. Circulation 1992;86:513-21.
} 